\title{
PARTICLE ACCELERATION SCALINGS BASED ON EXACT ANALYTIC MODELS FOR MAGNETIC RECONNECTION
}

\author{
I. J. D. CRAIG \\ Department of Mathematics, University of Waikato, Private Bag 3105, Hamilton, New Zealand; \\ i.craig@waikato.ac.nz \\ AND \\ YURI E. LITVINENKO \\ Institute for the Study of Earth, Oceans, and Space, University of New Hampshire, Durham, NH 03824-3525; \\ yuri.litvinenko@unh.edu \\ Received 2001 November 9; accepted 2002 January 13
}

\begin{abstract}
Observations suggest that particle acceleration in solar flares occurs in the magnetic reconnection region above the flare loops. Theoretical models for particle acceleration by the reconnection electric field, however, employ heuristic configurations for electric and magnetic fields in model current sheets, which are not solutions to the MHD reconnection problem. In the present study, particle acceleration is discussed within the context of a self-consistent MHD reconnection solution. This has the advantage of allowing poorly constrained local parameters in the current sheet region to be expressed in terms of the boundary conditions and electric resistivity of the global solution. The resulting acceleration model leads to energy gains that are consistent with those for high-energy particles in solar flares. The overall self-consistency of the approach is discussed.
\end{abstract}

Subject headings: acceleration of particles — MHD — Sun: activity — Sun: flares — Sun: magnetic fields

\section{INTRODUCTION}

Particle acceleration is an important signature of energy release in solar flares. Electrons with energies above $20 \mathrm{keV}$, which produce the flare hard X-rays and gamma rays via bremsstrahlung, may contain as much as $10 \%$ of the flare energy, up to $10^{31}$ ergs. The bulk of accelerated protons have energies within the range $0.1-10 \mathrm{MeV}$, and their energy content can exceed $10^{30}$ ergs (for a review, see Miller et al. 1997).

The current consensus is that flare energy is released through rapid dissipation of magnetic energy in the corona by virtue of magnetic reconnection (Priest \& Forbes 2000). The most promising geometry for flare energy release and particle acceleration, consistent with numerous observations in both impulsive and gradual flares, is that of a largescale reconnecting current sheet in the cusp region above soft X-ray flare loops (e.g., Aschwanden 1998; Metcalf \& Alexander 1999). Rapid reconnection in a large-scale current sheet is associated with a strong (super-Dreicer) electric field in the corona, which provides the most direct way to accelerate particles in flares. Much effort has gone into investigating charged-particle acceleration by the direct current (DC) electric field in the reconnecting current sheet (Speiser 1965; Martens 1988; Zhu \& Parks 1993; Litvinenko \& Somov 1993; Litvinenko 1996, 1997). Many important properties of the acceleration process, however, remain to be understood.

Most discussions of particle acceleration in reconnecting current sheets assume heuristic models for the electric and magnetic fields, which can describe only the immediate vicinity of the magnetic neutral point. Moreover, additional assumptions are required to define the local values of the fields and hence to calculate the properties of accelerated particles. On the other hand, numerically computed field configurations (e.g., Schopper, Birk, \& Lesch 1999) have limited applicability given the extremely high electric conductivity in the solar corona. The new generation of magnetic reconnection solutions, however, provide analytic description at arbitrary plasma resistivities and thus make it possible to achieve a more self-consistent approach in both two and three dimensions (Craig et al. 1995; Craig \& Henton 1995). In particular, the parameters of the particle acceleration region can be determined unambiguously using the exact global reconnection solution.

The purpose of this paper is to discuss the particle acceleration capabilities of the simplest magnetic reconnection solution available to us (Craig \& Henton 1995; Fabling \& Craig 1996). The model has one ignorable coordinate and can be used to model separator reconnection as well as strictly two-dimensional merging. Since particle acceleration timescales are so short, little error is involved in adopting a simplified steady-state description. The present discussion concentrates on deriving analytic scalings of particle energies with electric resistivity and complements the recent numerical work of Heerikhuisen et al. (2002). The basic mechanisms of current sheet particle acceleration are summarized in $\S 2$, while $\S 3$ describes the steady-state reconnection solution. Implications for particle acceleration, as well as the overall self-consistency of the approach, are discussed in $\S 4$.

\section{PARTICLE ORBITS IN THE RECONNECTING CURRENT SHEET}

\subsection{Introduction}

The problem of charged-particle motion in a current sheet is greatly simplified by the fact that typical acceleration lengths and timescales under solar flare conditions are very small compared to typical global parameters. This is why the usual approach in acceleration models is to approx- 
imate the reconnecting magnetic field by the first nonzero terms in the Taylor expansion inside the sheet:

$$
\boldsymbol{B}=-\left(\frac{y}{a}\right) B_{s} \hat{\boldsymbol{x}}-B_{\perp} \hat{\boldsymbol{y}}+B_{\|} \hat{\boldsymbol{z}} .
$$

Here the minus signs correspond to the electric current in the positive $z$-direction, and $a$ is the half-thickness of the sheet located at $y=0$. The expansion includes not only the reconnecting component of the magnetic field $B_{x}=B_{x}(y)$ but also the two nonreconnecting components $B_{\|}$and $B_{\perp}$ that are parallel and perpendicular to the plane of the sheet.

A nonzero $B_{\|}$corresponds to the regime of separator reconnection (Priest \& Forbes 2000; Litvinenko \& Craig 2000). Otherwise the field describes locally a two-dimensional current sheet. The reconnection electric field inside the sheet runs parallel to the current density $4 \pi \boldsymbol{J}=c \boldsymbol{\nabla} \times \boldsymbol{B}$, and so we may take $\boldsymbol{E}=E \hat{\boldsymbol{z}}$. Both $E$ and the nonreconnecting component $B_{\|}$can be assumed to be locally constant. Since the length scale of particle acceleration in the sheet is small, the spatial dependence of the field lines can be ignored, with the exception of the variation of $B_{x} \sim y / a$ across the current sheet thickness $2 a$. Hence, the perpendicular field $B_{\perp}$ is assumed to be constant on a given particle orbit.

The fact that $\boldsymbol{E} \cdot \boldsymbol{B} \neq 0$ indicates the presence of a significant resistive term in Ohm's law, required for magnetic reconnection:

$$
\boldsymbol{R}=\boldsymbol{E}+\frac{1}{c} \boldsymbol{v} \times \boldsymbol{B},
$$

where $v$ is the reconnection flow velocity and $c$ is the speed of light.

Even under the simplifying assumption $B_{\perp}=$ const, the character of the charged-particle motion for various relative values of the magnetic field components in the current sheet is nontrivial. In the limit $B_{\perp}=0$, whether $B_{\|}=0$ or not, the motion consists of the acceleration along the electric field $\boldsymbol{E}=E \hat{\boldsymbol{z}}$ and finite oscillations along the $y$-axis caused by the Lorentz force $\sim v_{z} B_{x}$ (Speiser 1965; Zhu \& Parks 1993). This idealized, highly symmetric situation, however, is unlikely to occur. In fact, any sheet model requires a nonzero $B_{\perp}$ as a result of reconnection itself (Martens 1988).

\subsection{Motion with Finite $B_{\perp}$}

Assume for the moment that the parallel field $B_{\|}$is small enough to be ineffective. Then the maximum displacement $l_{\text {acc }}$ along the electric field and the energy gain $\mathscr{E}$ are determined by the particle gyroradius in the perpendicular field $B_{\perp}$ :

$$
\mathscr{E}=2 m c^{2}\left(\frac{E}{B_{\perp}}\right)^{2}
$$

where $m$ is the particle mass (Speiser 1965). The acceleration time is of the order of the gyroperiod,

$$
t_{\mathrm{acc}}=\frac{\pi m c}{e B_{\perp}} .
$$

During this time, the initial downward motion of the particle is deflected through $90^{\circ}$ by the Lorentz force, and the particle achieves the speed $V=2 c E / B_{\perp}$.

Since the magnetic field in the solar corona is known to have a significant axial component along the coronal loops, the parallel field $B_{\|}$is likely to be on the order of the main reconnecting field $B_{s}$ at the edge of the sheet. A sufficiently strong parallel field magnetizes the particles and makes them follow the field lines. This can undo the gyrotying due to $B_{\perp}$ and lead to much stronger particle acceleration.

\subsection{Acceleration of Magnetized Particles}

When the magnetizing field is sufficiently strong, $B_{\|}>B_{\|, c}$, there is a transition to a new kind of motion in which particles move mainly along the field lines. The critical field is given by

$$
B_{\|, c}=\left(\frac{m c^{2} E B_{s}}{e a B_{\perp}}\right)^{1 / 2},
$$

where $m$ and $e$ are the particle mass and electric charge (Litvinenko 1996). This condition is easy to understand physically. If $B_{\|}=0$, particle orbits in the sheet are unstable, and the destabilizing Lorentz force component is given by

$$
F \simeq \frac{1}{c} e V B_{s}=\frac{B_{s}}{B_{\perp}} e E
$$

assuming some typical particle speed $V=c E / B_{\perp}$. The destabilization timescale is of the order

$$
t_{\perp}=\left(\frac{a m}{F}\right)^{1 / 2} .
$$

The instability becomes suppressed if it has no time to develop. Noting that $t_{\|}$is the timescale introduced by the parallel field,

$$
t_{\|}=\frac{m c}{e B_{\|}}
$$

we recover equation (5) by setting $t_{\perp}=t_{\|}$.

When the particle becomes magnetized in the sheet, its trajectory is no longer unstable. Although electrons are magnetized efficiently by the parallel field $B_{\|}$in the sheet, the effect is much harder to achieve for protons, since $B_{\|, c} \sim m^{1 / 2}$. In any case, the extra acceleration due to the magnetizing effect will cease when the particles leave the sheet. Integrating the magnetic field line equations defines the acceleration length $l_{\mathrm{acc}}$ as the displacement $\delta z$ along the electric field, which corresponds to $|\delta y|=a$ when the magnetized particles initially inside the sheet at $y=0$ leave the sheet along the field lines $l_{\text {acc }}=a B_{\|} / B_{\perp}$. The corresponding energy gain for the magnetized particles

$$
\mathscr{E}=\frac{B_{\|}}{B_{\perp}} e E a
$$

and the acceleration time

$$
t_{\text {acc }}=\left(\frac{B_{\|}}{B_{\perp}} \frac{2 a m}{e E}\right)^{1 / 2}
$$

are the main results of the local analytic approach to DC electric field acceleration in current sheets (Litvinenko 1996).

\subsection{Discussion}

The structure of the magnetic field inside the sheet has important consequences for particle orbits. Particle escape is generally much more efficient across the sheet than along it. If particles could simply move along the electric field 
direction through the total current sheet length, their typical energy would be the runaway energy given by the total potential drop $e U \simeq e E l$, where $l$ is the length scale of the current sheet. The effect of the perpendicular field component $B_{\perp}$ is to decrease the average acceleration length. If $\mathscr{E} \ll e U$, the electric field acceleration becomes a local acceleration mechanism that can occur throughout the reconnection region. We should remember, however, that the presence of a parallel, magnetizing component $B_{\|}$could in principle lead to acceleration lengths comparable to $l$, if $B_{\|} \simeq B_{\perp} l / a$.

The results of this section rely on the assumption that charged-particle orbits in the sheet are completely collisionless. Coulomb collisions can indeed be ignored for typical particle energies and acceleration times implied by solar flare observations. The role of plasma turbulence, however, is more important. Theoretical models predict the sheet thickness to be so small that the electron drift speed $u$ can exceed the threshold for a current-driven instability. Ionsound waves in particular are excited when $u$ exceeds the ion sound speed $c_{s}$, and the ion-sound turbulence is thought to be the primary factor that determines the magnitudes of transport coefficients in the sheet and hence its parameters (e.g., Somov 1992).

Thus, fast particles should be expected to generate plasma waves in the current sheet, and the waves in turn should scatter the particles and decrease the acceleration efficiency. The situation is formally analogous to the problem of charged-particle runaway in a DC electric field. Although a self-consistent solution to the problem is beyond the scope of this paper (see $\S 4.3$ ), it is clear that only the particles that are fast enough to overcome the friction force due to collisions can be accelerated by the electric field to high energies. Therefore, wave-particle interactions determine the fraction of particles entering the acceleration process, although they cannot influence the orbits and resulting energy gains of fast particles.

Even if we assume that the local description is sufficient to estimate the energies of flare particles, there still remains the difficulty that the heuristic configuration employed above is not a solution to a global reconnection problem. In particular, the critical dependence of the local $\boldsymbol{E}$ and $\boldsymbol{B}$ field components on the electric resistivity and boundary values remains unknown. In the remainder of this paper, we use an exact MHD solution for magnetic reconnection in order to specify the local parameters $a, E$, and $B_{\perp}$, and thus obtain more reliable estimates for the energies of particles accelerated in current sheets during solar flares.

\section{THE RECONNECTION MODEL}

\subsection{Analytic Reconnection Solutions}

Exact reconnection solutions can be developed within an incompressible MHD framework by superposing some small-scale disturbance fields $\boldsymbol{Q}(\boldsymbol{r})$ onto a global background field $\boldsymbol{P}(\boldsymbol{r})$. The explicit construction is

$$
\boldsymbol{v}=\alpha \boldsymbol{P}+\frac{\beta}{\alpha} \boldsymbol{Q}, \quad \boldsymbol{B}=\beta \boldsymbol{P}+\boldsymbol{Q},
$$

where $\alpha$ defines the amplitude and $\beta$ the shear of the velocity field. The planar solution of Craig \& Henton (1995) provides the prototype for this approach (Fig. 1).

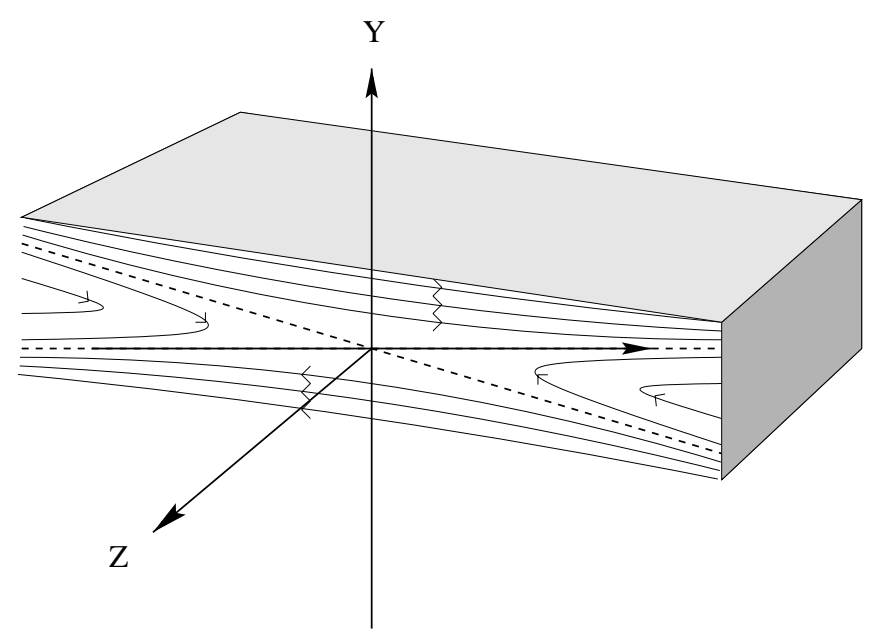

FIG. 1.-Current sheet geometry in the magnetic reconnection solution. Dotted lines show separatrices of the field. Note that one separatrix - the one with no flow across it - is contiguous with the $x$-axis running along the sheet. Particles gyrocaptured in the sheet are ejected through the outer edges $|x|=1$.

The exact solution is also naturally extended to describe separator (2.5-dimensional) reconnection as well as spine and fan reconnection at three-dimensional magnetic nulls (Craig et al. 1995). It is also possible to incorporate timedependent inertial terms and Hall current contributions in Ohm's law, a point we mention in $\S 3.3$.

Let us express the solution in terms of the reference coronal values

$$
B_{c}=10^{2} \mathrm{G}, \quad \ell_{c}=10^{9.5} \mathrm{~cm}, \quad v_{\mathrm{A}}=10^{9} \mathrm{~cm} \mathrm{~s}^{-1},
$$

based on a preflare number density of $10^{9} \mathrm{~cm}^{-3}$. The solution that encompasses both the plane and the separator reconnection (Craig et al. 1995; Fabling \& Craig 1996) is given by

$$
\begin{gathered}
\boldsymbol{v}=\alpha x \hat{\boldsymbol{x}}-\alpha y \hat{\boldsymbol{y}}-\frac{\beta}{\alpha} \boldsymbol{Q}(y), \\
\boldsymbol{B}=\beta x \hat{\boldsymbol{x}}-\beta y \hat{\boldsymbol{y}}-\boldsymbol{Q}(y),
\end{gathered}
$$

where

$$
\begin{gathered}
\boldsymbol{Q}=\frac{E}{\eta \mu} \operatorname{Daw}(\mu y) \hat{\boldsymbol{x}}-B_{\|} \hat{\boldsymbol{z}}, \\
\mu^{2}=\frac{\alpha^{2}-\beta^{2}}{2 \alpha \eta}, \quad \operatorname{Daw}(x)=\int_{0}^{x} \exp \left(t^{2}-x^{2}\right) d t .
\end{gathered}
$$

Unlike the local heuristic model of the previous section, the expressions above fully describe the magnetic and velocity fields in the volume, without extraneous parameterization. Note that the exact MHD solution leads to an electric field aligned to the $z$-axis only when the nonreconnecting magnetic field component $B_{\|}$vanishes. In other words, only when $B_{\|}=0$ can we assume a uniform electric field throughout the reconnection region

$$
\boldsymbol{E}=E \hat{\boldsymbol{z}} .
$$

Even if $B_{\|} \neq 0$, the plane components of the electric field vanish at the singular point $x=y=0$. In what follows, we 
consider only the effect of the dominant $z$-component of the electric field.

It is important to stress that the collisional plasma resistivity $\eta$ is very small, as measured by an inverse Lundquist number of the order of $10^{-14}$ in the present dimensionless formulation. Significant reconnection rates can therefore take place only if the disturbance field $Q$ is strongly localized. We must take $\alpha>|\beta|$ to achieve such small length scales, and additional constraints are required to obtain limitations on the merging rate. One difficulty is that the amplitude of the flow field is decoupled from the amplitude of the pile-up field. This deficiency can be remedied-invoking pressure continuity as in Sweet-Parker merging - by ensuring that material exhausted from the sheet is determined Alfvénically by strength of the current layer (Litvinenko \& Craig 1999, 2000). Even so, the pressure of the sheet must eventually saturate, since the solution has to match to external "driving regions" outside the reconnection region.

Once the reconnection solution is adjusted using the pressure continuity and saturation arguments outlined above, the reference parameters given in equation (12) imply a total electric current in the sheet of the order of $10^{12} \mathrm{~A}$. This value is in agreement with both theoretical models for energy accumulation and release on the Sun (e.g., Somov 1992) and coronal large-scale currents deduced from vector magnetograms (e.g., Metcalf et al. 1994). We return to this point when discussing the self-consistency of the particle acceleration model in $\S 4.3$.

\subsection{Resistive Scaling of the Current Layer}

It is now understood that when saturation of the current layer is imposed, the pile-up reconnection solution allows well-defined merging rates to be determined (Craig \& Watson 2000; Litvinenko \& Craig 2000). Let us express the properties of the resulting current sheet in terms of the peak field in the current layer $B_{s}$ so that, for example, $\alpha=\max |\boldsymbol{Q}| \equiv B_{s}$.

The current layer is conveniently defined as the region $y \leq\left|y_{s}\right|$ sandwiched between the peaks of the flux pile-up disturbance field $|\boldsymbol{Q}|$. The Dawson function $\operatorname{Daw}(\mu y)$ peaks when $\mu y_{s} \simeq 1$, and if we assume, to achieve concrete scalings, the typical value $\beta=\alpha / \sqrt{2}$, we find that $\mu^{2}=\alpha /(4 \eta)$. The half-width $a$ of the sheet is accordingly

$$
a \equiv y_{s}=2\left(\frac{\eta}{B_{s}}\right)^{1 / 2} \quad\left[\ell_{c}\right] \text {. }
$$

Note that, for clarity and convenience, we adopt the practice of augmenting dimensionless equations with conversion factors (in square brackets) required to recover conventional units (cgs). The inflow speed to the sheet is just $\alpha y_{s}$, and so

$$
v_{\text {in }}=2\left(\eta B_{S}\right)^{1 / 2} \quad\left[v_{\mathrm{A}}\right] .
$$

By contrast, the exhaust flow is Alfvénic

$$
v_{\text {out }}=B_{s} \quad\left[v_{\mathrm{A}}\right] .
$$

It is straightforward to use these results to deduce the ohmic dissipation rate

$$
W=\frac{1}{2} \eta^{1 / 2} B_{s}^{5 / 2} \quad\left[v_{\mathrm{A}} \frac{B_{c}^{2}}{8 \pi} \ell_{c}^{2}\right]
$$

and the magnitude of the reconnection electric field,

$$
E=\frac{1}{2} \eta^{1 / 2} B_{s}^{3 / 2} \quad\left[\frac{v_{\mathrm{A}} B_{c}}{c}\right]
$$

As we shall repeatedly emphasize, the electric field is strongly super-Dreicer for all plausible resistivities $\eta$. The geometry of the current layer (see Fig. 1) shows that the field running perpendicular to the sheet has the strength

$$
B_{\perp}=\sqrt{2} \eta^{1 / 2} B_{S}^{1 / 2} \quad\left[B_{c}\right] .
$$

This is actually the perpendicular field strength at the outer edge $(x=1)$ of the layer. The perpendicular field at some intermediate point $x$ is simply $B_{y}(x)=B_{\perp} x / \ell_{c}$.

\subsection{Hall Current Effects}

Given the likely breakdown of collisional conditions, it might be suspected that extra contributions should be included in equation (2) that defines the reconnection electric field. Of various possible contributions, the Hall current term $\boldsymbol{E}_{\mathrm{H}}$ is most likely to be significant (see Priest \& Forbes 2000 and references therein).

Including $\boldsymbol{E}_{\mathrm{H}}$ modifies the Ohm's law as

$$
\boldsymbol{E}+\frac{\boldsymbol{v} \times \boldsymbol{B}}{c}=\eta \boldsymbol{J}+\frac{\boldsymbol{J} \times \boldsymbol{B}}{n e c},
$$

ignoring the electron pressure gradient for simplicity. The last term becomes comparable to $\eta J$ when the normalized resistivity is small enough:

$$
\eta<\frac{c}{\ell_{c} \omega_{p}} \frac{B}{B_{c}}
$$

where $\omega_{p}=\left(4 \pi n e^{2} / m_{p}\right)^{1 / 2}$ is the proton plasma frequency. This criterion for the importance of $\boldsymbol{E}_{\mathrm{H}}$ is very robust; it depends primarily on the local reconnection magnetic field $\boldsymbol{B}(\boldsymbol{r})$, since all other factors derive from the preflare reference values given by equation (12). Recall that, in supplying the flare power, magnetic fields of several hundred $\mathrm{G}$ are required to provide electric currents in excess of $10^{12} \mathrm{~A}$ (see eq. [38]). The assumed reference coronal values suggest that Hall currents must be important for $\eta<10^{-7}$, that is, for all collisional resistivities of interest. In order to demonstrate Hall current effects, numerical simulations should be based on sufficiently low resistivities (Bhattacharjee, Ma, \& Wang 1999).

To what extent does a generalized Ohm's law affect the reconnection model we adopt? A detailed analysis is beyond the scope of the present study, but we can readily check that the form (eq. [11]) of the reconnection solution goes through when inertial effects and Hall current contributions are included. The main modification due to $\boldsymbol{E}_{\mathrm{H}}$ is the presence of additional planar components in the electric field accompanied by nonuniformities in the axial field $\boldsymbol{B}_{\|}$. A strictly two-dimensional description is no longer permissible, but, as with the present model, the strength of the axial field can still be set independently.

As far as particle acceleration is concerned, the typical energy change due to the Hall electric field scales as $\int \boldsymbol{E}_{\mathrm{H}} \cdot \boldsymbol{d} \boldsymbol{r} \simeq a E_{\mathrm{H}} \sim B_{s}^{2}$ independent of $\eta$. Hence, the Hall current effect on the particle energies is not determined by resistivity. Moreover, for the merging solution at hand, the reconnection field $\boldsymbol{E}$ in the sheet is determined primarily by 
the $z$-component of the induction equation. In contrast, $\boldsymbol{E}_{\mathrm{H}} \sim \boldsymbol{J} \times \boldsymbol{B}$ is strictly planar, and it vanishes at the neutral point $x=y=0$. It follows that although Hall current effects are likely to modify general magnetic merging models, they do not appear to compromise the estimates for typical particle energies that assume a uniform electric field in the $z$ direction. The additional planar components of the $\boldsymbol{E}_{\mathrm{H}}$ field could, however, modify the spectrum of accelerated particles. Particle drift into the sheet could also be affected, as well as the self-consistency requirements of the hybrid particle/MHD model (§ 4.3).

\subsection{Turbulent Merging and Effective Resistivity}

Suppose for the moment that a classical collisional resistivity $\eta \simeq 10^{-14}$ can be adopted. Then the ohmic dissipation rate given by equation (21) is rather weak, $W \simeq 10^{26}$ ergs $\mathrm{s}^{-1}$, even for quite significant flux pile-up solutions, say, $B_{s} \simeq 10$. Yet the electric field, although modest, $E \simeq 10^{-5}$ cgs, still exceeds by 2 orders of magnitude the Dreicer field,

$$
E_{\mathrm{D}}=\frac{4 \pi n e^{3} \ln \Lambda}{k T} \simeq 10^{-7} \mathrm{cgs},
$$

based on $T \simeq 2 \times 10^{6} \mathrm{~K}$ and $\ln \Lambda \simeq 20$. The implication is that the current density is unrealistically high. In fact, $J \simeq n e c$, whereas a more plausible limit would probably involve the ion sound speed $c_{s}$ rather than the speed of light.

More specifically, equation (18) predicts that the thickness of the current layer is $y_{s} \simeq 2 \times 10^{2} \mathrm{~cm}$ if the resistivity remains collisional. This value, however, is 4 orders of magnitude less than the collisional mean free path based on coronal parameters in equation (12) and-assuming a preflare proton thermal speed of the order of $10^{7} \mathrm{~cm} \mathrm{~s}^{-1}$-at least 2 orders below the thermal proton gyroradius associated with the perpendicular field $B_{\perp}$ within the sheet. All these factors suggest that a collisional resistivity is not appropriate within the current layer.

The need for a modified resistivity is confirmed by the following simple calculation. If we assume that the current density predicted by the analytic model, namely $J=E / \eta$ with $E$ given by equation (22), cannot exceed the limit $J^{*}=n e u^{*}$ for some $u^{*}<c$, we obtain

$$
\frac{B_{s}^{3 / 2}}{\eta^{1 / 2}} \frac{B_{c}}{8 \pi \ell_{c}}<n e \frac{u^{*}}{c} .
$$

This reduces to $\eta^{1 / 2}>10^{-8.5} B_{s}^{3 / 2}\left(c / u^{*}\right)$ for the reference parameters in equation (12). Identifying $u^{*}$ with the ion sound speed, typically $c_{s} \simeq 10^{-3} c$, suggests that, even for modest flux pile-up factors, effective resistivities must satisfy $\eta>10^{-9}$ to achieve realistic current densities. The need for an enhanced resistivity in fact goes through for any $u^{*}<c$.

Current-limited turbulent resistivity is capable of providing a more plausible super-Dreicer merging solution (Litvinenko \& Craig 2000). The anomalous resistivity $\eta_{a} \sim E$ has the desired effect of limiting the current density to $J \simeq 10^{8}$ (cgs), a value comparable to the ion sound speed for a coronal electron temperature $T_{e} \simeq 10^{7}-10^{8} \mathrm{~K}$, all other parameters being preflare parameters in equation (12). The net effect is to thicken up the sheet and enhance the ohmic dissipation rate to a level exceeding $10^{28} \mathrm{ergs} \mathrm{s}^{-1}$. For the present parameters and flux pile-up factors approaching $B_{s} \simeq 10$, these results can be made consistent with the collisional formulae of $\S 3.2$ by adopting an effective resistivity of magnitude

$$
\eta_{\text {eff }} \simeq 10^{-8} .
$$

With these assumptions, the merging solution can be used as a convenient analytic platform for exploring particle acceleration by the reconnection electric field. In particular, the parameters underlying the heuristic current sheet of $\S 2$ can be identified. These confirm that particle acceleration times are at least 2 orders of magnitude below Alfvénic merging times.

Note that the model for anomalous resistivity assumes the electric current density to be determined by electrons drifting with speeds of order $u=J / n e$. The electric force acting on these particles is balanced by the effective friction force due to turbulent waves. However, as argued in $\S 2.4$, a certain fraction of particles $D$ will run away and gain much larger energies defined by the collisionless estimate (eq. [9]). This fraction is not easy to determine because the magnitudes of the accelerating electric field and the turbulent ana$\log$ of the Dreicer field are of the same order, as indicated by the fact that $u \approx c_{s}$. Although the determination of $D$ and the associated contribution to the total electric current is necessary for self-consistent treatment of reconnection, the results of this paper remain valid as long as $D \ll 1$.

To obtain concrete numbers, we assume a moderate flux pile-up factor $B_{s}=7$. The previous current sheet formulae now give results broadly consistent with the turbulent model of Litvinenko \& Craig (2000): $W \simeq 10^{28} \mathrm{ergs} \mathrm{s}^{-1}$, $E=10^{-2.5}$ (cgs), $B_{\perp}=0.04 \mathrm{G}, y_{s}=2 \times 10^{5} \mathrm{~cm}$, and $v_{\text {in }}=10^{5.5} \mathrm{~cm} \mathrm{~s}^{-1}$. For definiteness, we assume these values in exploring particle acceleration spectra.

\section{PARTICLE ACCELERATION BASED ON THE ANALYTIC RECONNECTION MODEL}

\subsection{Phases of Particle Acceleration}

The MHD solution described in the previous section contains very strong magnetic fields external to the current sheet. Particles must escape the constraints of gyrotying before they can be significantly accelerated by the reconnection $\boldsymbol{E}$ field. This can happen only in the region close to the neutral point where the magnetic field is either weak or coaligned with the electric field. Therefore, only a very small part of reconnection volume $\ell_{c}^{3}$ can participate directly in the acceleration. In this region, we expect the effects of both the Hall current and the plane electric field to be minor because of the smallness of $\boldsymbol{J} \times \boldsymbol{B}$.

There are in fact three distinct phases associated with particle acceleration (Heerikhuisen et al. 2002). Consider first the two-dimensional magnetic field configuration with $B_{\|}=0$ (Speiser 1965). In the initial phase, particles gyrotied in the field of the advection region drift into the sheet with speed $\left(\mathrm{cm} \mathrm{s}^{-1}\right)$

$$
V_{d}=c \frac{E}{B_{s}} .
$$

Strong acceleration occurs in the second phase for particles in the weak field region close to the neutral point. The $z$ acceleration allows the Lorentz force of the reconnecting field $\sim B_{x} V_{z}$ to become effective. Gyrocapture eventually 
occurs at the speed

$$
V=2 c \frac{E}{B_{y}},
$$

where the acceleration length is the gyroradius $R_{g}=m c v /\left(e B_{y}\right)$. After capture, the particle can drift away through the edge $|x|=1$ of the sheet.

The description is similar in the case of separator reconnection with $B_{\|}>B_{\|, c}$ (Litvinenko 1996), although now the magnetized particles effectively follow the guiding magnetic field throughout the volume. The burst of acceleration along the $z$-axis occurs near the singular point where the electric and magnetic fields are co-aligned, followed by the drift phase out of the sheet.

In considering the distinct phases of the particle acceleration, we should remember that the perpendicular field $B_{y}$ varies linearly with distance $x$ from the neutral point. This means that particles injected at different $x$-coordinates experience different values of $B_{y}$ and achieve different energies. Particles that experience sufficiently weak $B_{y}$ escape capture altogether. It is this inhomogeneity that gives rise to variations in acceleration length and accounts for particle energy spectra.

\subsection{Particle Energies}

In the nonrelativistic approximation, particles captured by $B_{\perp}$ achieve the energy $\mathscr{E}=m V^{2} / 2$. Since uncaptured particles achieve the limit $\mathscr{E}=e E \ell_{c}$, which remains valid in the relativistic case, we obtain the upper and lower energy bounds for the two-dimensional current sheet,

$$
2 m c^{2}\left(\frac{E}{B_{\perp}}\right)^{2} \leq \mathscr{E} \leq e E \ell_{c} .
$$

In the case of accelerated protons, these bounds give

$$
10 \mathrm{MeV} \leq \mathscr{E}_{p} \leq 3 \mathrm{GeV} .
$$

Note that since captured electrons achieve the same speed as protons, capture energies should be reduced by a factor of $m_{e} / m_{p} \simeq 1840$ when considering electron spectra. Hence, captured electrons range upward from $5 \mathrm{keV}$.

As indicated above, a simple continuity argument shows that the inhomogeneity in $B_{y}(x)$ results in a power-law energy distribution, specifically, $B_{y}(x) \sim x$ leads to $\sim \mathscr{E}^{-3 / 2}$. Test particle simulations confirm that the result is not sensitive to details of magnetic geometry (e.g., Mori, Sakai, \& Zhao 1998) and even to the inclusion of relativistic effects (Heerikhuisen et al. 2002). The robust shape of the predicted spectrum suggests that a more detailed self-consistent treatment is required in order to explain typically observed softer distributions in solar flares. Polarization electric fields in the sheet due to charge separation can also modify the spectrum.

$\mathrm{GeV}$ energies can also be reached by invoking a strong field $B_{\|}$. The critical magnetizing field $B_{\|, c}$ is of the order of $50 \mathrm{G}$ for protons and only $1 \mathrm{G}$ for electrons. The resulting energies

$$
\mathscr{E}=\frac{B_{\|}}{B_{\perp}} e E a
$$

can be as high as the total potential drop $\simeq 3 \mathrm{GeV}$ along the sheet, assuming that the magnetizing field $B_{\|}$can be of the same order as the reconnecting field $B_{s}$. However, it should be remembered that very efficient acceleration may result in unrealistically large electric currents, necessitating a more self-consistent treatment. Physically, the magnetic field associated with the particle current becomes large enough to disturb the MHD solution, hence requiring its modification (see $\S 4.3)$.

The use of an exact reconnection model for the field structure makes it possible to determine scalings for particle energy gains, which depend only on the electric resistivity, the flux pile-up factor, and the reference boundary values. Specifically, we have in dimensionless form

$$
\mathscr{E} \sim \eta^{0} B_{s}^{2}
$$

if the parallel magnetic field $B_{\|}<B_{\|, c}$ and

$$
\mathscr{E} \sim \eta^{1 / 2} B_{S}^{1 / 2}
$$

if $B_{\|}>B_{\|, c}$. Note that the energy gain is independent of resistivity in the two-dimensional case, so the energy is determined only by the flux pile-up factor - a result that derives from the fact that the ratio $E / B_{\perp}$ is independent of $\eta$ in the reconnection solution. Remarkably, even with this constraint, the strictly two-dimensional case seems quite favorable as far as particle energies and acceleration spectra are concerned. In all cases, the upper cutoff is determined by the strength of the electric field, hence the scaling $\mathscr{E}_{\max } \simeq e E \ell_{c} \sim \eta^{1 / 2} B_{s}^{3 / 2}$ for the maximum particle energy.

The ability of our approach to predict analytic scalings of particle energies with resistivity and magnetic field demonstrates the advantage of using exact reconnection models in particle acceleration studies. In the case of magnetized particles, it should be remembered that the energy gain given by equation (33) cannot exceed and, from self-consistency requirements, should be on average less than the total potential drop $\simeq e E \ell_{c}$ that bounds all energy gains for particles accelerated along the length of the current sheet.

\subsection{Consistency Constraints}

It is important to check that the proton and electron currents implied by the acceleration formulae are consistent with the MHD reconnection model. Consider the twodimensional current sheet for simplicity. First note that the drift velocity into the sheet is comparable to the bulk inflow speed in equation (19),

$$
V_{d}=c \frac{E}{B_{S}} \simeq v_{\text {in }} \simeq 2 \times 10^{5} \mathrm{~cm} \mathrm{~s}^{-1} .
$$

A similar consistency condition should hold for the outflow,

$$
V=2 c \frac{E}{B_{\perp}} \simeq v_{\text {out }},
$$

where $v_{\text {out }}$ is the exhaust speed of material ejected from the sheet (e.g., Martens \& Young 1990). Since $v_{\text {out }}$ is just the Alfvén speed of the flux pile-up current layer, we have that $v_{\text {out }} \simeq 7 \times 10^{9} \mathrm{~cm} \mathrm{~s}^{-1}$. This value is perfectly consistent with the particle speed $V$.

A recurring difficulty with DC electric field acceleration models is the strength of the electric current associated with the particle beam. The total current through the sheet fol- 
lows from Ampere's law:

$$
I_{s}=\frac{c}{4 \pi} \int \boldsymbol{B} \cdot \boldsymbol{d l} \simeq \frac{c}{2 \pi} B_{s} \ell_{c} \simeq 4 \times 10^{12} \mathrm{~A} .
$$

This estimate must be consistent with the flux of accelerated particles, which is clearly

$$
\mathscr{N} \simeq 2 n_{c} v_{\text {in }} \ell_{c}^{2} \simeq 4 \times 10^{33} \mathrm{~s}^{-1} .
$$

Most of these particles are in fact captured within the sheet. The beam current at any $z$-slice due to protons (ignoring the contribution from much more easily captured electrons) is therefore

$$
I_{\text {cap }}=\mathscr{N} e D,
$$

where $D \simeq l_{\text {acc }} / \ell_{c}$ is a dilution factor that accounts for the fact that a typical accelerated proton traverses only a small fraction of the total $z$-length before being captured as well as for a finite probability of overcoming the turbulent friction force. Compatibility with equation (38) requires $D \leq 10^{-2}$, which seems plausible given that the lowest energy protons have gyroradii of the order of $10^{-3} \ell_{c}$.

It is worth stressing that $D \ll 1$ appears to be necessary in order to reconcile the electric currents based on Ampere's law and the flux of accelerated particles. In fact, the question is more general. It is well known, for example, that if flare energy is released in the form of a high-energy electron beam, the beam-related electric current will exceed the MHD current by several orders of magnitude (Knight \& Sturrock 1977). On the other hand, there is not much freedom in the parameters of the sheet $B_{s}$ and $\ell_{c}$ that would result in a larger current through the sheet. This is why it would be difficult to obtain a highly anisotropic distribution of high-energy particles in any reconnection model. We avoid this problem in our model because a short acceleration length leads to $D \ll 1$, and thus the emerging particle flux is spread over a large area of the current sheet rather than concentrated into a dense beam.

Even in the two-dimensional case, however, about $10 \%$ of particles are not captured - and these are effectively free streaming. The free-streaming condition is simply $B_{y}(x)<E$ (Alekseyev \& Kropotkin 1970), which corresponds to particles close enough to the neutral point, roughly $x / \ell_{c}<0.1$. The presence of a magnetizing component only worsens the problem by increasing particle energies. Reducing the number flux (eq. [39]) by an order of magnitude still yields a proton-carried current that exceeds the MHD current (eq. [38]) by more than a factor of 5 . Therefore, despite the encouraging agreement of the outflow and inflow constraints, there remain some doubts as to the overall self-consistency of the approach.

\section{DISCUSSION AND CONCLUSIONS}

Analytical studies of the charged-particle motion and acceleration in reconnecting current sheets have demonstrated very interesting effects associated with the magnetic field structure in the sheet. Local values of the field components within the weak field regions of the current sheet determine the character of particle orbits and the acceleration efficiency. It should be stressed, however, that only detailed reconnection solutions, involving global velocity and magnetic fields, can determine the local $\boldsymbol{E}$ and $\boldsymbol{B}$ fields in a self-consistent manner. In this paper, we demonstrate explicitly how analytic reconnection solutions can be used to infer unambiguously the parameters of the reconnecting current sheet and hence the properties of the emerging spectrum of accelerated particles.

We have pointed out that even the simplest MHD reconnection solution of Craig \& Henton (1995), involving strictly two-dimensional fields, seems well suited to rapid energy release and significant particle acceleration (see also Heerikhuisen et al. 2002). The reconnection electric field is highly super-Dreicer, and the long, thin current sheet allows strong acceleration for a significant number of particles. The sheet geometry means that gyrocapture by the perpendicular field component $B_{\perp}$ is relatively mild, allowing acceleration to occur over appreciable distances. A nontrivial property of the two-dimensional solution is that the final energy of captured particles is independent of the plasma resistivity $\eta$ (eq. [34]). For typical coronal parameters, electron energies range upward from several $\mathrm{keV}$ to the runaway limit of a few GeV. Protons are harder to capture, and so the lower cutoff for protons is predicted to be of the order of several $\mathrm{MeV}$. The form of the energy spectrum derives from spatial inhomogeneities in the perpendicular field component and approximates a negative power law of exponent $3 / 2$ if energy-loss processes are completely ignored - an assumption to be relaxed in future studies.

The presence of a significant parallel component $\boldsymbol{B}_{\|}$, coaligned with the nonplanar $\boldsymbol{E}$ field, can undo gyrocapture and significantly increase acceleration energies. One artifact of the magnetizing effect is an increase in the number of runaway particles - a problem that exacerbates the self-consistency of the particle/MHD approach. Recall that, even in the two-dimensional case, the number of runaway protons leads to a particle current that seems to be too great by about an order of magnitude. Self-consistency requires that the reconnection current (eq. [38]) should dominate the particle current. The latter can only be strengthened by the magnetizing field, and there is no way to overrule the separator model as a valid MHD solution. This may mean that the analytic reconnection solutions at hand can describe magnetic reconnection on the Sun only if the field pile-up factor $B_{s}$ is not too large. Alternatively, the proton flux should decrease if only a fraction of particles in the reconnection inflow eventually enters the acceleration process. Hence, either the particle model or the MHD solution may have to be modified to achieve a fully self-consistent solution.

Either way, it is clear that a successful flare model must explain both the total power output and the properties of accelerated particles in flares. The present exploratory approach does produce encouraging particle energies, and parallel numerical work by Heerikhuisen et al. (2002) shows that the results can be applied to timeextended proton acceleration in large gradual solar flares. Observations suggest that particles are energized in some gradual flares in a current sheet formed below a coronal mass ejection (Klein \& Trottet 2001). A more detailed treatment, however, is required to achieve a self-consistent particle/MHD model. One possibility is a full twofluid description for the reconnection field. Another approach - and this seems promising in terms of decreasing the strength of free-streaming particle current-is to examine three-dimensional reconnection solutions that involve neutral points rather than planar nulls. In this 
case, exact solutions are available that can be analyzed along the lines presented here (Craig et al. 1995). Again, observations strongly suggest that such three-dimensional null points are involved in active phenomena in the solar corona (Aulanier et al. 2000). In terms of a purely MHD description, however, there seems to be no compelling reason why neutral-point solutions should be preferred to separator reconnection models that incorporate strong magnetizing fields.

This work was partly supported by NSF grant ATM9813933, NASA grants NAG5-7792 and NAG5-8228, and Marsden Fund grant 96-UOW-MIS-0006. We are grateful to J. Heerikhuisen for numerous useful discussions.
Alekseyev, I. I., \& Kropotkin, A. P. 1970, Geomagnetism and Aeronomy, 10,755

Aschwanden, M. J. 1998, in Observational Plasma Astrophysics: Five Years of Yohkoh and Beyond, ed. T. Watanabe et al. (Dordrecht: Kluwer), 285

Aulanier, G., DeLuca, E. E., Antiochos, S. K., McMullen, R. A., \& Golub, L. 2000, ApJ, 540, 1126

Bhattacharjee, A., Ma, Z. W., \& Wang, X. 1999, J. Geophys. Res., 104, 14543

Craig, I. J. D., Fabling, R. B., Henton, S. M., \& Rickard, G. J. 1995, ApJ, $455, \mathrm{~L} 197$

Craig, I. J. D. \& Henton, S. M. 1995, ApJ, 450, 280

Craig, I. J. D., \& Watson, P. G. 2000, Sol. Phys., 194, 251

Fabling, R. B., \& Craig, I. J. D. 1996, Phys. Plasmas, 3, 2243

Heerikhuisen, J., Litvinenko, Y. E., \& Craig, I. J. D. 2002, ApJ, 566, 512

Klein, K. L.. \& Trottet, G. 2001, Space Sci. Rev., 95, 215

Knight, J. W., \& Sturrock, P. A. 1977, ApJ, 218, 306

\section{EFERENCES}

Litvinenko, Y. E. 1996, ApJ, 462, 997 1997, Phys. Plasmas, 4, 3439

Litvinenko, Y. E., \& Craig, I. J. D. 1999, Sol. Phys., 189, 315 2000, ApJ, 544, 1101

Litvinenko, Y. E., \& Somov, B. V. 1993, Sol. Phys., 146, 127

Martens, P. C. H. 1988, ApJ, 330, L131

Martens, P. C. H., \& Young, A. 1990, ApJS, 73, 333

Metcalf, T. R., \& Alexander, D. 1999, ApJ, 522, 1108

Metcalf, T. R., et al. 1994, ApJ, 428, 860

Miller, J. A., et al. 1997, J. Geophys. Res., 102, 14631

Mori, K., Sakai, J., \& Zhao, J. 1998, ApJ, 494, 430

Priest, E. R. \& Forbes, T. G. 2000, Magnetic Reconnection: MHD Theory and Applications (Cambridge: Cambridge Univ. Press)

Schopper, R., Birk, G. T., \& Lesch, H. 1999, Phys. Plasmas, 6, 4318

Somov, B. V. 1992, Physical Processes in Solar Flares (Dordrecht: Kluwer)

Speiser, T. W. 1965, J. Geophys. Res., 70, 4219

Zhu, Z., \& Parks, G. 1993, J. Geophys. Res., 98, 7603 